\title{
Integrative transcriptomic and proteomic analysis reveals mechanisms of silica-induced pulmonary fibrosis in rats
}

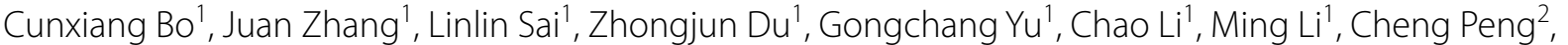 \\ Qiang Jia ${ }^{1 *}$ and Hua Shao ${ }^{1^{*}}$
}

\begin{abstract}
Background: Silicosis is a systemic disease characterized by persistent inflammation and incurable pulmonary fibrosis. Although great effort has been made to understand the pathogenesis of the disease, molecular mechanism underlying silicosis is not fully elucidated. This study was aimed to explore proteomic and transcriptomic changes in rat model of silicosis.

Methods: Twenty male Wistar rats were randomly divided into two groups with 10 rats in each group. Rats in the model group were intratracheally instilled with $50 \mathrm{mg} / \mathrm{mL}$ silicon dioxide $(1 \mathrm{~mL}$ per rat) and rats in the control group were treated with $1.0 \mathrm{~mL}$ saline $(1 \mathrm{~mL}$ per rat). Twenty-eight days later, transcriptomic analysis by microarray and tandem mass tags (TMT)-based proteomic analysis were performed to reveal the expression of mRNAs and proteins in lung tissues. Gene Ontology (GO) and Kyoto Encyclopedia of Genes and Genomes (KEGG) were applied to analyze the altered genes and proteins. The integrated analysis was performed between transcriptome and proteome. The data were further verified by RT-qPCR and parallel reaction monitoring (PRM).

Results: In total, 1769 differentially expressed genes (DEGs) and 650 differentially expressed proteins (DEPs) were identified between the silicosis model and control groups. The integrated analysis showed 250 DEPs were correlated to the corresponding DEGs (cor-DEPs-DEGs), which were mainly enriched in phagosome, leukocyte transendothelial migration, complement and coagulation cascades and cellular adhesion molecule (CAM). These pathways are interrelated and converged at common points to produce an effect. GM2a, CHI3L1, LCN2 and GNAl1 are involved in the extracellular matrix (ECM) and inflammation contributing to fibrosis.
\end{abstract}

Conclusion: Our comprehensive transcriptome and proteome data provide new insights into the mechanisms of silicosis and helpful information for more targeted prevention and treatment of silicosis.

Keywords: Silicosis, Proteomics, Transcriptomics, $\mathrm{SiO}_{2}$, Rat

*Correspondence: jiaqiang5632@163.com; chinashaohua5888@163.com

1 Shandong Academy of Occupational Health and Occupational Medicine, Shandong First Medical University \& Shandong Academy of Medical Sciences, Ji'nan, Shandong, China

Full list of author information is available at the end of the article

\section{Background}

Silicosis is a systemic disease characterized by persistent inflammation and progressive pulmonary fibrosis. The disease is caused by long-term inhalation of occupational dust containing silicon dioxide [1]. Once onset, even if the exposure is terminated, silicosis can continue to develop and result in breath difficulty, low blood oxygen levels and eventual death [2,3]. It is well established 
that the pathological process of silicosis involves phagocytosis, inflammation, extracellular matrix (ECM) remodeling, epithelial-mesenchymal transition (EMT) and fibrosis. However, the exact molecular mechanism underlying the silicosis is not fully elucidated and there are no specific drugs that can effectively alleviate or reverse silica-induced lung injury. Therefore, there is an urgent need to undersand the molecules and molecular pathways leading to silicosis.

Several distinct and complementary techniques have the potential to yield new perspectives on understanding the molecular activities and functions $[4,5]$. Transcriptional profiling using high-throughput RNA sequencing technology is a useful method for identifying novel transcripts and analyzing gene expression changes in pathophysiology and toxicology [6, 7]. While gene expressions and their regulations are important in biological functions, protein expression represents outcomes of interaction between genetic and non-genetic factors and provides phenotype information of a disease [8]. Therefore, joint analysis of transcriptomic and proteomic data from the same samples can provide more comprehensive information on molecular mechanisms of a disease.

Silica particle-induced pulmonary fibrosis in rat model has been widely used in silicosis studies. In this study, we evaluated key target genes and proteins and associated pathways involved in silicosis through an integrative analysis with mRNA microarray and tandem mass tags (TMT) data. This comprehensive analysis of the transcriptome and proteome may substantially improve the overall understanding of the potential molecular mechanisms in silicosis.

\section{Methods \\ Study protocol}

This study was approved by the Committee on the Ethics of Animal Experiments of Shandong Academy of Occupational Health and Occupational Medicine (Protocol Number: 20190003). Twenty specific-pathogenfree (SPF) male Wistar rats (160-180 g) were purchased from Beijing Vital River Laboratory Animal Technology Co., Ltd. (Beijing, China), and housed in an SPF facility at $22 \pm 2 \mathrm{C}, 55 \% \pm 10 \%$ relative humidity with a $12 \mathrm{~h}$ light/dark cycle and free access to water and chow. After one week, the rats were allocated to two groups with the random number table: the model group $(\mathrm{n}=10)$ and the control group $(\mathrm{n}=10)$, which were intratracheally instilled with $50 \mathrm{mg} / \mathrm{mL}$ silicon dioxide $(1 \mathrm{~mL}$ per rat) and normal saline solution $(1 \mathrm{~mL}$ per rat) respectively. Rats in the same group were housed together with five rats per cage. The individual rat was considered the experimental unit in this study. All rats were sacrificed on day 28 after administration. Please refer to our previous work for the detailed procedures of establishment and evaluation of rat silicosis model [9]. Based on the histological changes, three lungs from each group were selected for microarray and TMT assay to achieve the minimum requirements for biological replicates. All rats were intratracheally instilled with silicon dioxide under sodium pentobarbital anesthesia and sacrificed by carbon dioxide anesthesia after the exposure.

\section{Microarray assay}

Total RNA was extracted using TRIzol Reagent (Cat\# 15596-018, Life Technologies, Carlsbad, CA, USA) according to the manufacturer's instructions and quantified by the NanoDrop ND-2000 (Thermo Scientific). The RNA integrity was assessed using Agilent Bioanalyzer 2100 (Agilent Technologies). An mRNA microarray analysis was conducted on each total RNA sample using a Low Input Quick Amp Labeling Kit, One-Color (Cat\# 5190-2305, Agilent Technologies) and a Gene Expression Hybridization Kit (Cat\# 5188-4242, Agilent Technologies) following the manufacturer's protocol. Slides were scanned using an Agilent Microarray Scanner (Cat\# G2505C, Agilent Technologies) with the default settings. Feature Extraction software 10.7 (Agilent Technologies) was used to evaluate the raw datas which were normalized using the Quantile algorithm, Gene Spring Software 14.8 (Agilent Technologies).

\section{RT-qPCR assay}

The lung tissues for RT-qPCR assay were same to the microarray assay. Total RNA was extracted as described above and double-strand cDNA was synthesized using the HiScript II Q RT SuperMix for qPCR (+gDNA wiper) Kit (R223-01, Vazyme, Nanjing, China) according to the manufacturer's instructions. RT-qPCR was performed using ChamQ SYBR qPCR Master Mix Kit (Q311-03, Vazyme, Nanjing, China) on LightCycler ${ }^{\circledR}$ 480 II Real-time PCR Instrument (Roche, Swiss). The primers were synthesized by Generay Biotech (Generay, PRC) based on the mRNA sequences obtained from the NCBI database (Table 1). The expression levels of

Table 1 Primer sequences

\begin{tabular}{lll}
\hline Gene & Forward primer & Reverse primer \\
\hline CHI3L1 & CCAATCACAGGGTCAGGATTA & TGAGGAAGTCGCATATCTCGTA \\
LCN2 & CCGATGAACTGAAGGAGC & GTCGGTGGGAACAGAGAA \\
GM2a & TGTACGCCATGCTCCTTC & CCAGCTTGAACTTAAGATTCCA \\
GNAl1 & GGTTCTGTGTTTGGCAGT & AGTCTGTGCAACGTTTATACAA \\
\hline
\end{tabular}


mRNAs were normalized to ACTB and were calculated using the $2^{-\Delta \Delta \mathrm{Ct}}$ method [10].

\section{TMT and PRM assay}

Proteins from lung were extracted and digested, then the digested peptides were labeled using TMT Reagent-6plex Multiplex Kit according to the manufacturer's instructions (90066B, Thermo Scientific). LC-MS/MS was performed using a $\mathrm{Q}$ Exactive mass spectrometer (Thermo Scientific) combined with Easy nLC system 1200 (Thermo Scientific). The TMT labeling, peptide fractionation and mass spectrometer detection were carried out by Shanghai luming biological technology co., LTD (Shanghai, China). To examine the reliability of TMT mass spectrometry, four DEPs (GM2a, CHI3L1, LCN2 and GNAI1) were validated by parallel reaction monitoring (PRM) assay. Details of TMT and PRM assay were referred to our previous study [9].

\section{Bioinformatics analysis}

Transcriptomics and proteomics data were first analyzed using Statistical Program for Social Sciences (SPSS) (SPSS Inc., version 20.0, United States). A fold change $\geq 2.0$ and $P$ values $\leq 0.05$ was used to classify differentially expressed genes (DEGs). The significantly different expressed proteins (DEPs) were screened with a fold change $\geq 1.5$ and $P$ values $\leq 0.05$ by t-test, and a multiple testing correction was performed using Benjamini and Hochberg procedure to control the False Discovery Rate (FDR), using $P$ value $(<5 \%)$ [11]. Gene Ontology (GO) and Kyoto Encyclopedia of Genes and Genomes (KEGG) analysis were applied to determine the roles of these DEGs and DEPs, and the GO analysis was divided into biological process (BP), cellular component (CC) and molecular function (MF). $P$ values less than 0.05 were considered significantly enriched.

\section{Correlation analysis of transcriptomics and proteomics}

Genes are regulated at multiple levels during the expression process. At present, most studies have reported that the expression consistency between mRNAs and their corresponding proteins is not high enough. Therefore, we conducted a combined analysis of the proteome and transcriptome to gain a better understanding of the regulation of gene expression [12]. All expression data related to proteomics and transcriptomics were analyzed with the pearson correlation coefficient analysis between the model and control groups. The gene and its coding protein expressed with significant threshold of fold change and $P$ value were identified and named as cor-DEPsDEGs genes. The cor-DEPs-DEGs genes were further analyzed with GO and KEGG databases, and the spearman correlation coefficient was calculated.

\section{Results}

\section{Histopathological evaluation of lung tissue}

HE staining showed lung tissues were normal in control rats. In contrast, damaged alveolar structures, infiltrating inflammatory cells and silicotic nodule were observed in each rat from silica-treated group, which showed rat silicosis model was successfully replicated in this study. The detailed histological changes were referred to our previous study [9].

\section{DEGs and bioinformatics analysis}

In total, 1769 DEGs were differentially expressed between the model and control groups. 952 DEGs were significantly upregulated, and 817 DEGs were significantly downregulated (Additional file 1: Table S1). These DEGs were enriched in 753 GO terms. The BP category was associated with inflammatory response, response to lipopolysaccharide and angiogenesis (Fig. 1a). The MF category was associated with extracellular space, extracellular exosome and cell surface (Fig. 1b). The CC category was associated with identical protein binding, protein homodimerization activity and chemokine activity (Fig. 1c). KEGG analysis proved these DEGs were enriched in 58 pathways, including complement and coagulation cascades, chemokine signaling pathway, cytokine-cytokine receptor interaction, Rap1 signaling pathway and Toll-like receptor signaling pathway (Table 2).

\section{DEPs and bioinformatics analysis}

A total of 650 DEPs were identified in rats from the model and control groups (Additional file 2: Table S2). Among them, 355 proteins were significantly upregulated, and 295 proteins were significantly downregulated. These DEPs were enriched in 1967 GO terms. The BP category was associated with cell adhesion, biological adhesion and cellular response to chemical stimulus. The MF category was associated with cell adhesion molecule binding, cadherin binding and protein binding. The CC category was associated with extracellular region part, extracellular vesicle and extracellular organelle (Fig. 2). KEGG analysis proved these DEPs were enriched in 40 pathways, including collecting duct acid secretion, ribosome, phagosome, lysosome, leukocyte transendothelial migration, glycosaminoglycan degradation, glutathione metabolism and ECM-receptor interaction (Table 3).

\section{Protein-mRNA correlation analysis}

Integration analysis of above proteomic and transcriptomic data was conducted. All identified mRNAs with DEPs were matched, followed by transformation of DEPs and transcript volume ratios into $\log 2$ forms. An investigation of changes at both the transcript and 


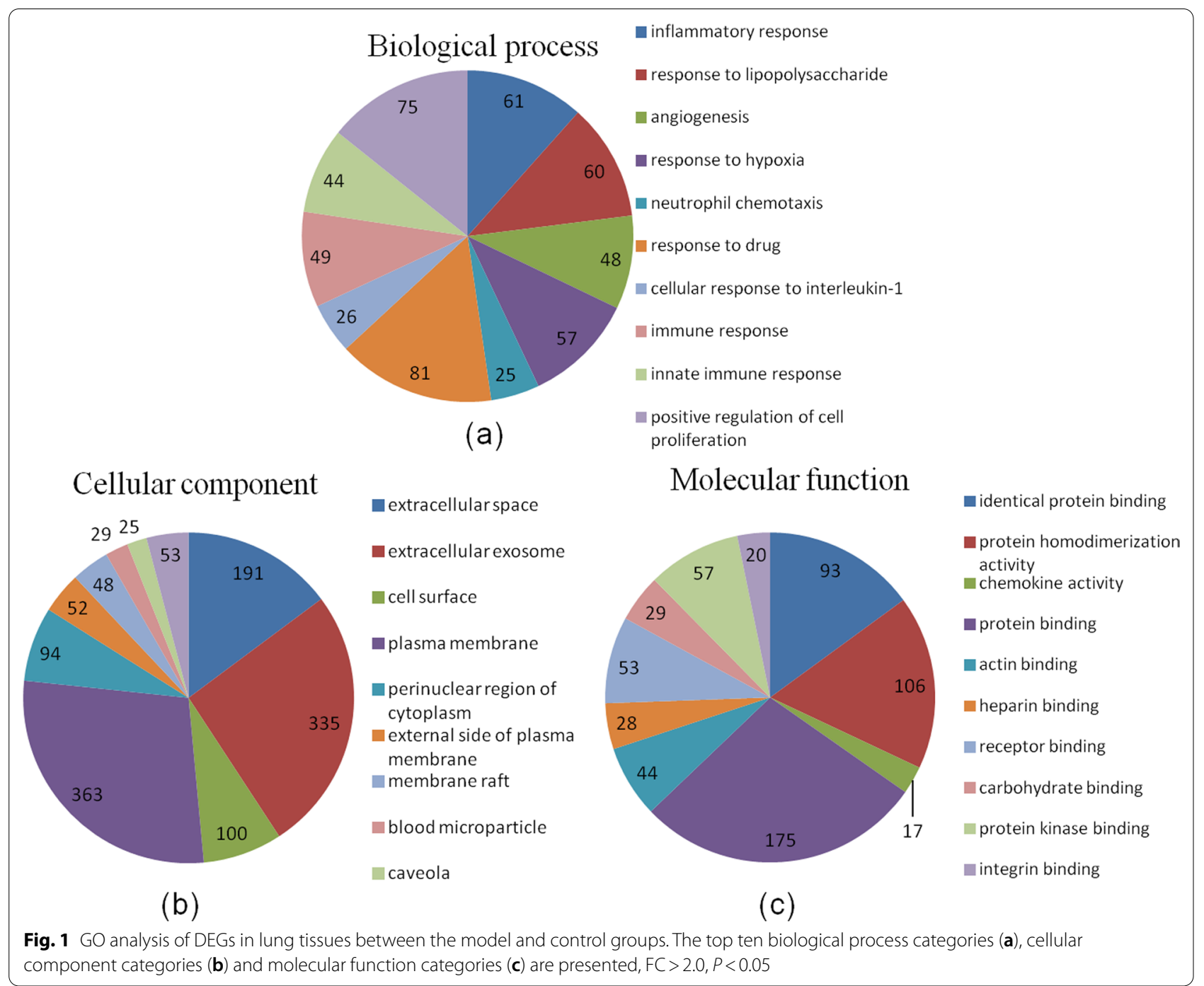

Table 2 The top ten KEGG pathways enrichment of DEGs in model rat lungs

\begin{tabular}{lll}
\hline Pathway ID & Description & P value \\
\hline rno04610 & Complement and coagulation cascades & $8.26 \mathrm{E}-10$ \\
rno05150 & Staphylococcus aureus infection & $6.11 \mathrm{E}-08$ \\
rno05323 & Rheumatoid arthritis & $1.27 \mathrm{E}-07$ \\
rno04062 & Chemokine signaling pathway & $1.00 \mathrm{E}-06$ \\
rno04060 & Cytokine-cytokine receptor interaction & $1.78 \mathrm{E}-06$ \\
rno04015 & Rap1 signaling pathway & $5.94 \mathrm{E}-06$ \\
rno05133 & Pertussis & $1.02 \mathrm{E}-05$ \\
rno04620 & Toll-like receptor signaling pathway & $2.53 \mathrm{E}-05$ \\
rno05140 & Leishmaniasis & $2.90 \mathrm{E}-05$ \\
rno04142 & Lysosome & $5.16 \mathrm{E}-05$ \\
\hline
\end{tabular}

tanslational levels revealed a relatively low correlation (Spearman correlation coefficient, $\mathrm{R}=0.668$, Fig. 3A) for all genes and proteins assessed. When we compared the 1769 DEGs with the 650 DEPs, the higher positive correlations were indicated $(\mathrm{R}=0.917$, Fig. $3 \mathrm{~B})$. When the correlation between DEGs and their corresponding DEPs with the same or opposite trend was analysed, higher positive and negative correlations were indicated ( $R=0.940$, Fig. $3 C$ and $R=-0.932$, Fig. 3D). Among the cor-DEGs-DEPs genes, 126 upregulated (Fig. 3E) and 118 downregulated genes (Fig. 3F) were identified as the same trend as protein expressions, while 6 genes had the opposite trend with their correlating proteins (Additional file 3: Table S3). These cor-DEGs-DEPs genes might play important roles in lung injuries of rats exposed to silica particles [13].

To further explore the potential functions of those cor-DEGs-DEPs genes in silicosis, GO terms and KEGG 


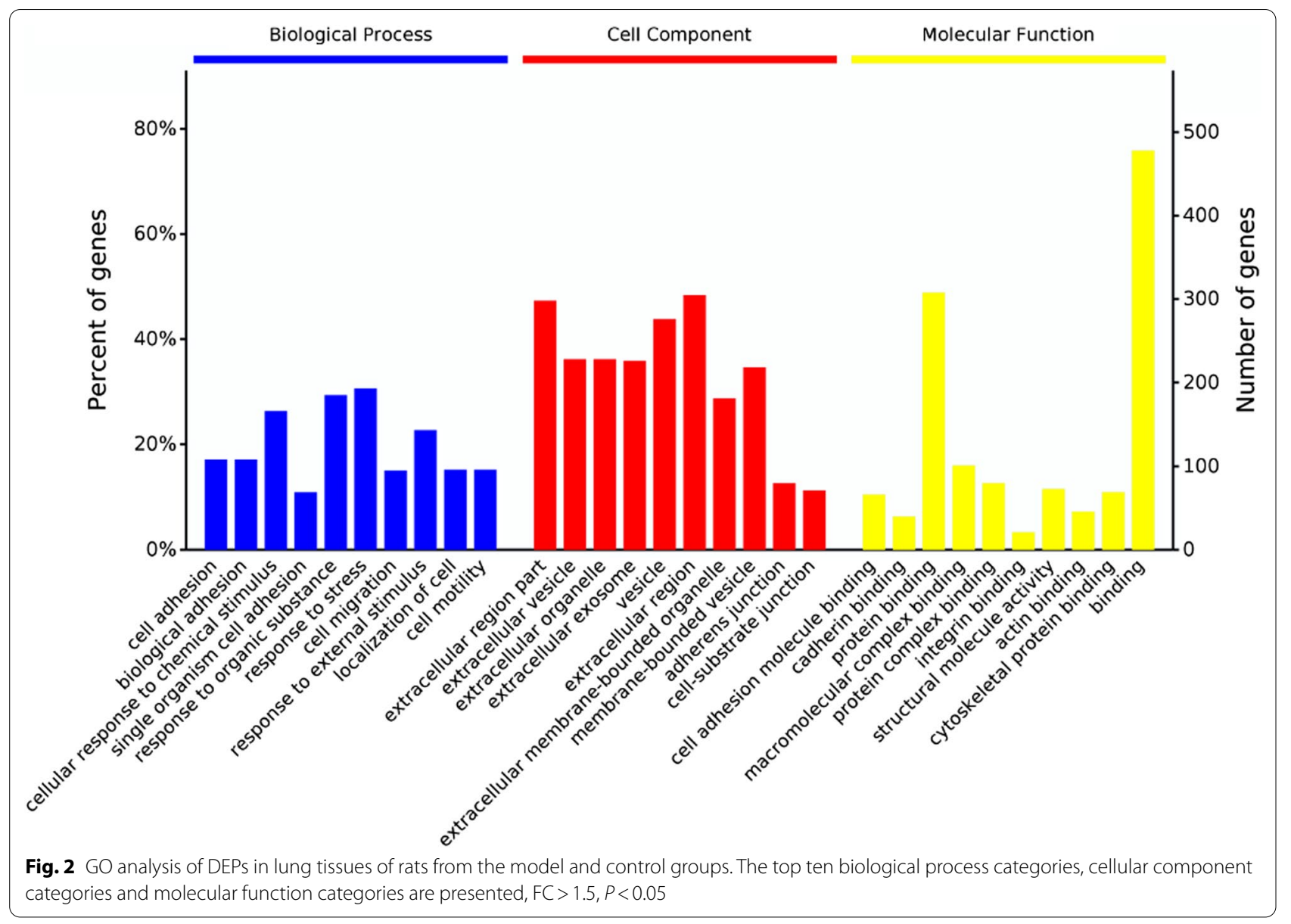

Table 3 The top ten KEGG pathways enrichment of DEPs in model rat lungs

\begin{tabular}{lll}
\hline Pathway ID & Description & P value \\
\hline rno04966 & Collecting duct acid secretion & $5.74 \mathrm{E}-09$ \\
rno03010 & Ribosome & $3.56 \mathrm{E}-08$ \\
rno04145 & Phagosome & $4.78 \mathrm{E}-07$ \\
rno04142 & Lysosome & $8.09 \mathrm{E}-07$ \\
rno04670 & Leukocyte transendothelial migration & $3.18 \mathrm{E}-05$ \\
rno00531 & Glycosaminoglycan degradation & $4.01 \mathrm{E}-05$ \\
rno00480 & Glutathione metabolism & 0.000107 \\
rno05323 & Rheumatoid arthritis & 0.000181 \\
rno04614 & Renin-angiotensin system & 0.000495 \\
rno04964 & Proximal tubule bicarbonate reclamation & 0.000584 \\
\hline
\end{tabular}

pathways were enriched at the levels of transcriptome and proteome, respectively. GO terms were highly enriched at both mRNA and protein levels (Fig. 4 and Additional file 4: Table S4). The subcategory identified in the BP category were cell differentiation, immune system process and primary metabolic process. For CC category, intracellular, extracellular exosome and proteincontaining complex were the most abundant categories. The most abundant MF were protein and ion binding. KEGG pathway analysis showed 16 pathways were highly enriched at both mRNA and protein levels, including phagosome, leukocyte transendothelial migration, complement and coagulation cascades and cell adhesion molecules (CAMs) (Fig. 5 and Additional file 5: Table S5).

\section{(See figure on next page.)}

Fig. 3 Correlations between the expression of proteins and genes. The horizontal-axis represents the protein expression level, and the vertical-axis represents the gene expression level. A Scatterplot of the relationship between genes identified in the transcriptome and proteome. B Scatterplot and correlation coefficients between DEGs and DEPs. C Scatterplot and correlation coefficients between DEGs and DEPs in the same trend. D Scatterplot and correlation coefficients between DEGs and DEPs in the opposite trend. E Venn diagram of upregulated DEGs and DEPs. F Venn diagram of downregulated DEGs and DEPs. Yellow part, DEGs; Red part, DEPs; Middle part, overlapped DEGs and DEPs 
(A)

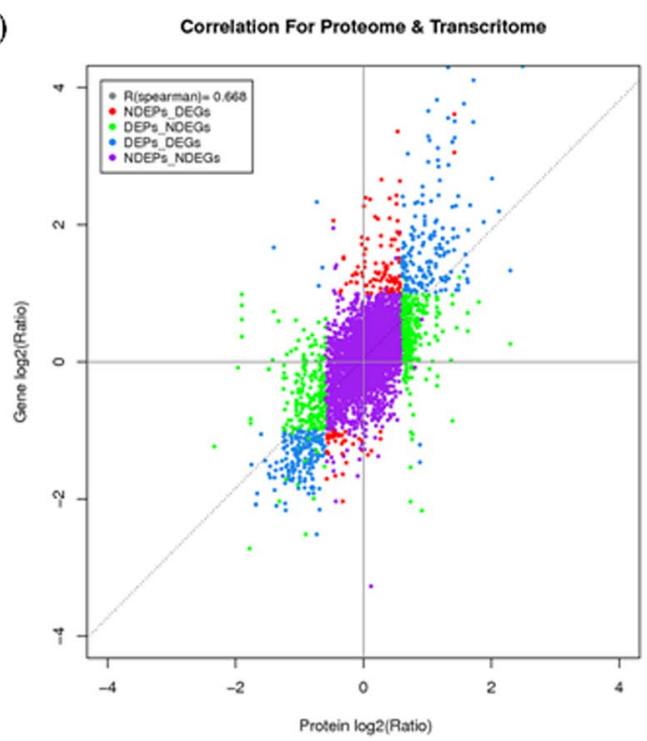

(C)

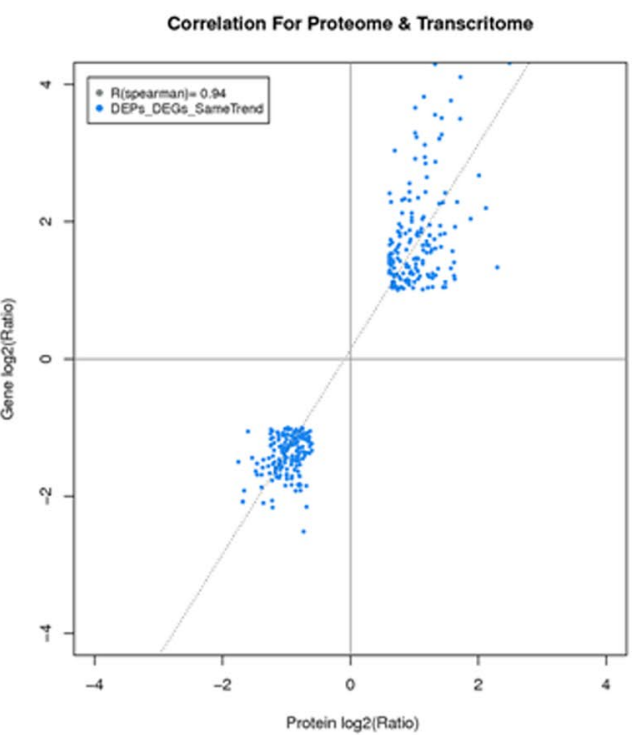

(E)

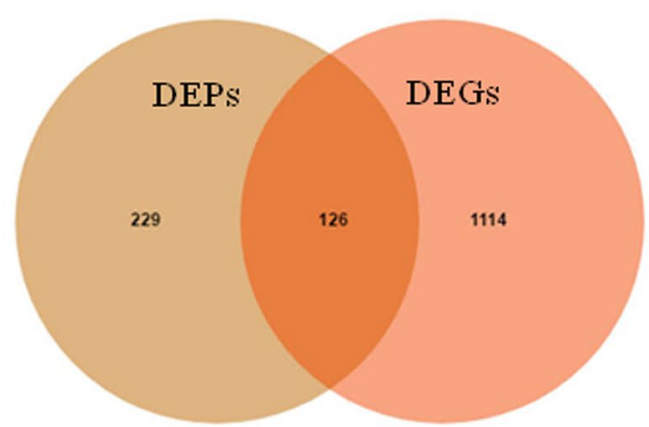

(B)

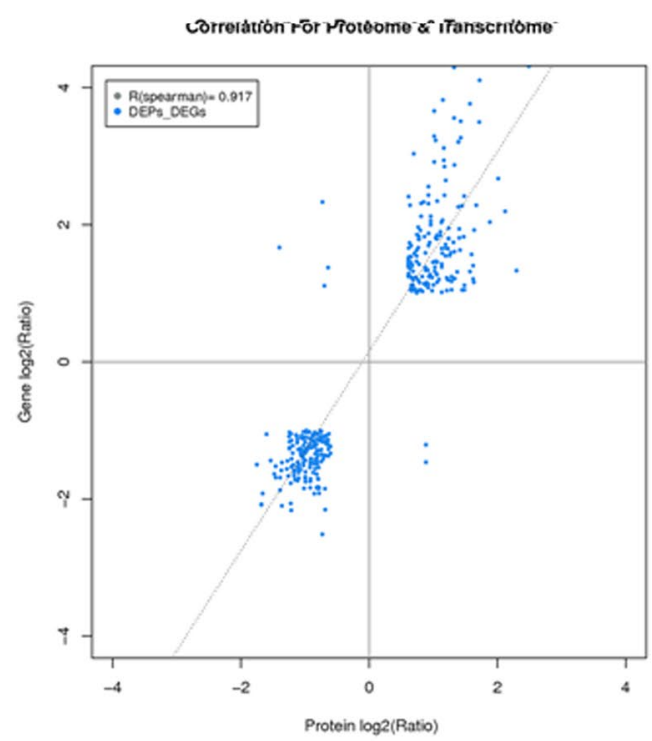

(D)

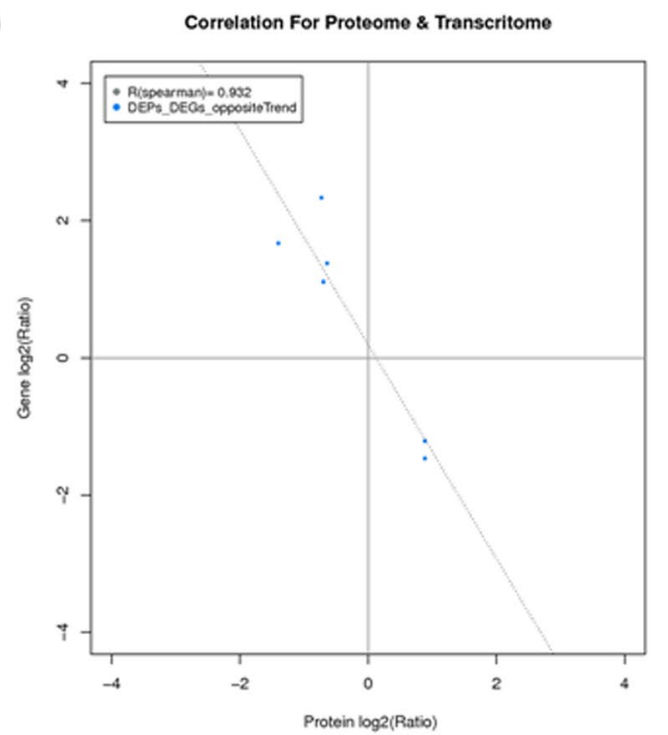

(F)

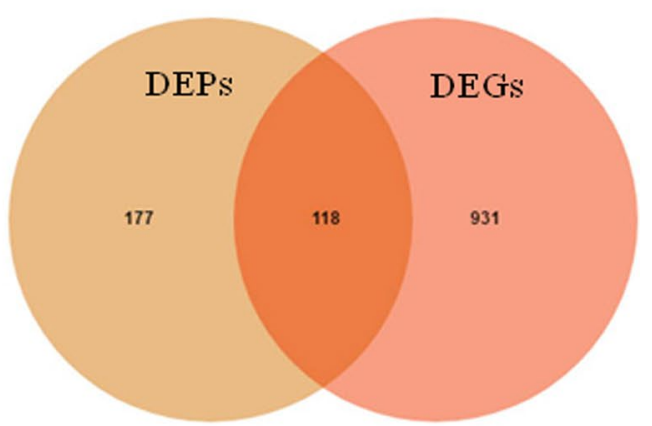

Fig. 3 (See legend on previous page.) 
(A)

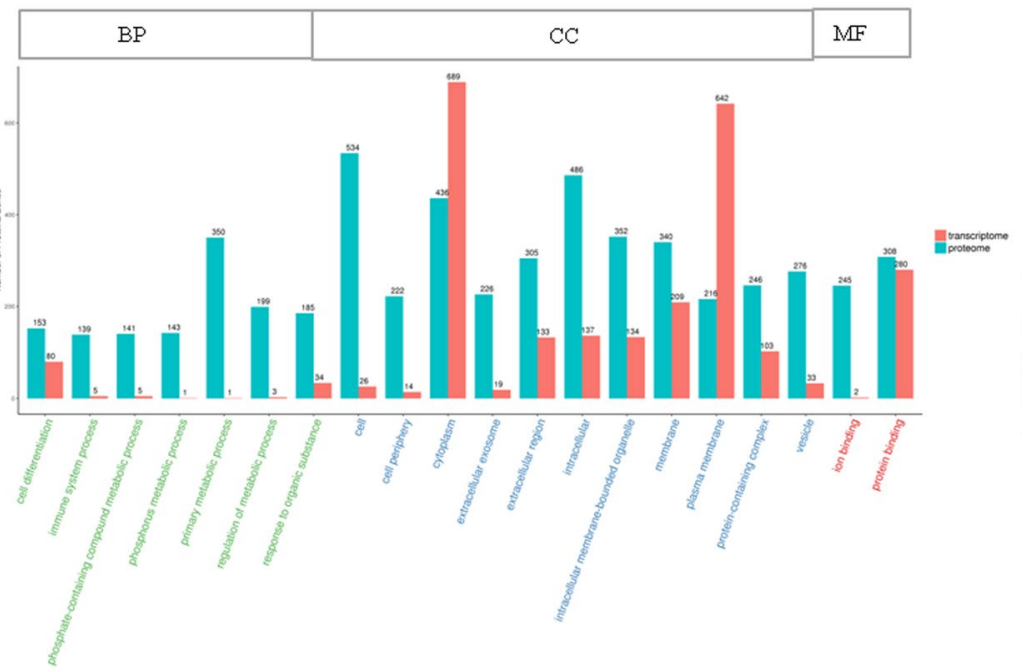

(B)

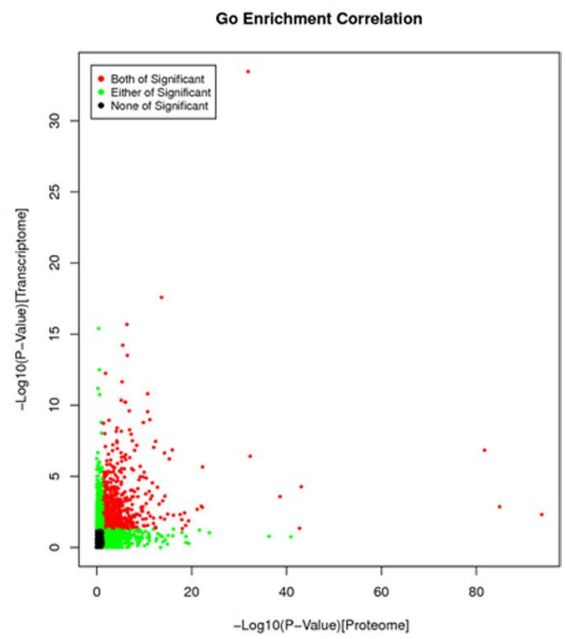

Fig. 4 Correlation of GO enrichment between transcriptome and proteome. A Number of GO enrichment correlation between proteome and transcriptome. Each column in the figure represents a GO secondary annotation entry, Green represents DEPs, and red represents DEGs. The top twenty enriched terms of DEPs are presented. BP: biological process, CC: cellular component, MF: molecular function. B The overview scatter diagram of $\mathrm{GO}$ enrichment correlation between the protein level and transcript level of genes

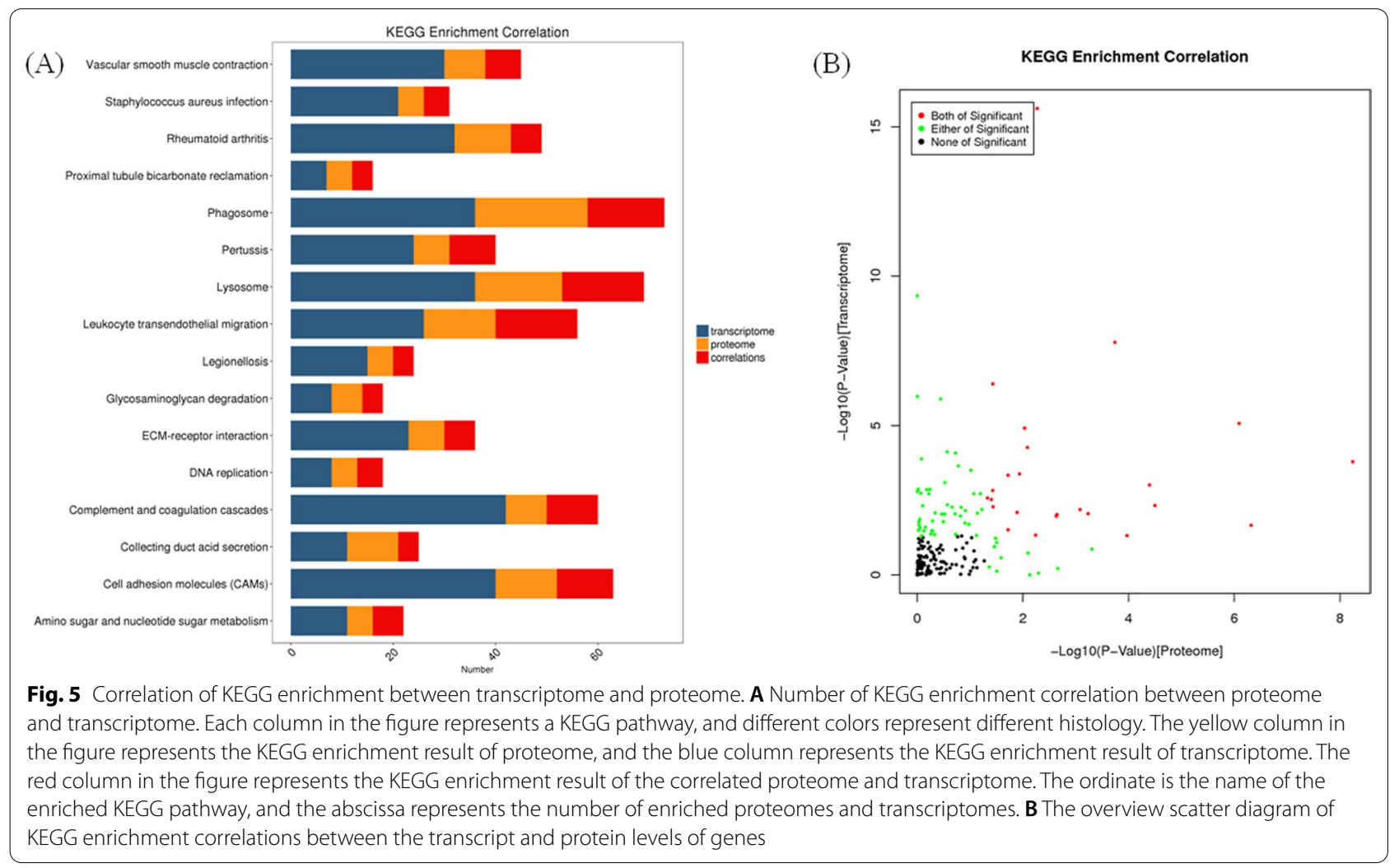




\section{Data verification of microarray and TMT}

To validate the reliability of the microarray data in this study, we selected four DEGs (upregulated GM2a, CHI3L1, LCN2 and downregulated GNAI1) for RT-qPCR assays. The results showed that all tested DEGs were significant changed between the model and control groups. The expression patterns were consistent with the results of microarray, although the folds of changes were different (Fig. 6A), which indicated that our transcriptome results might reflect the relative expression level of each gene in vivo. As shown in Fig. 6B, the three upregulated DEPs (GM2a, CHI3L1, LCN2) and downregulated GNAI1 were in the same trend as that observed when the protein levels were quantified by PRM, suggesting that the TMT data in this study was reliable. The difference of expression level (fold change) may be due to the different detection methods.

\section{Discussion}

The aim of the study was to explore the molecular mechanism underlying silicosis and try to identify potential biomarkers that can predict the pathological progress of silicosis. To this end, we investigated the DEGs and DEPs in the lungs of the rats exposed to silica particles by microarray chip technology and TMT-coupled LCMS/MS. The reliability of the results from microarray and TMT was confirmed by RT-qPCR and PRM assay. In total, 250 cor-DEPs-DEGs genes were identified in the lung tissues of rats from the silicosis model and control groups. Results of the KEGG pathway analysis indicated these cor-DEPs-DEGs genes were mainly enriched in phagosome, leukocyte transendothelial migration, complement and coagulation cascades and cellular adhesion molecule (CAM). For the first time, we found that cellular adhesion molecules (CAM), such as Gm2a, CHI3L1, LCN2, THBD, NADPH oxidase (NOXs) and vacuolarATPase are involved in pathogenesis of silicosis.

When the lung is injured, bleeding and leakage of plasma proteins from damaged surfaces in the alveolar space form a fibrinous deposit. Subsequently, the fibrin clot is invaded by proliferating fibroblasts, which forms the durable components of ECM [14]. Then the fibrin was degraded by plasmin. Decreased ECM degradation caused by deficient function of alveolar fibrinolysis plays an important role in the formation of pulmonary fibrosis in lung [15]. Excessive procoagulant and decreased fibrinolytic activities have been observed in animal models of lung fibrosis and pulmonary samples of patients with interstitial lung diseases [16, 17]. Procoagulant status in idiopathic pulmonary fibrosis (IPF) patients is associated with increased serum levels of factor VIII C, fibrinogen, homocysteine and protein $\mathrm{C}$ activity [18]. Thrombomodulin (THBD) plays an important role in anticoagulant by inducing plasminogen (PLG) to plasmin, which is inactivated by combining with $\mathrm{C} 3 \mathrm{~b}$ or CFH. Deletion of THBD can also cause abnormal activation of complement substitution pathway [19]. In this study we found that the downregulated THBD may result in the deficient of plasmin and ECM deposition. Fibrin and derivatives have been reported to exacerbate lung inflammation by stimulating the expression of leukocyte adhesion molecules, chemokines and proinflammatory cytokines [15]. Therefore, anticoagulant therapy could be helpful in silicosis treatment.

An imbalance of oxidation/antioxidation is a crucial mechanism of pulmonary fibrosis [20]. It has been
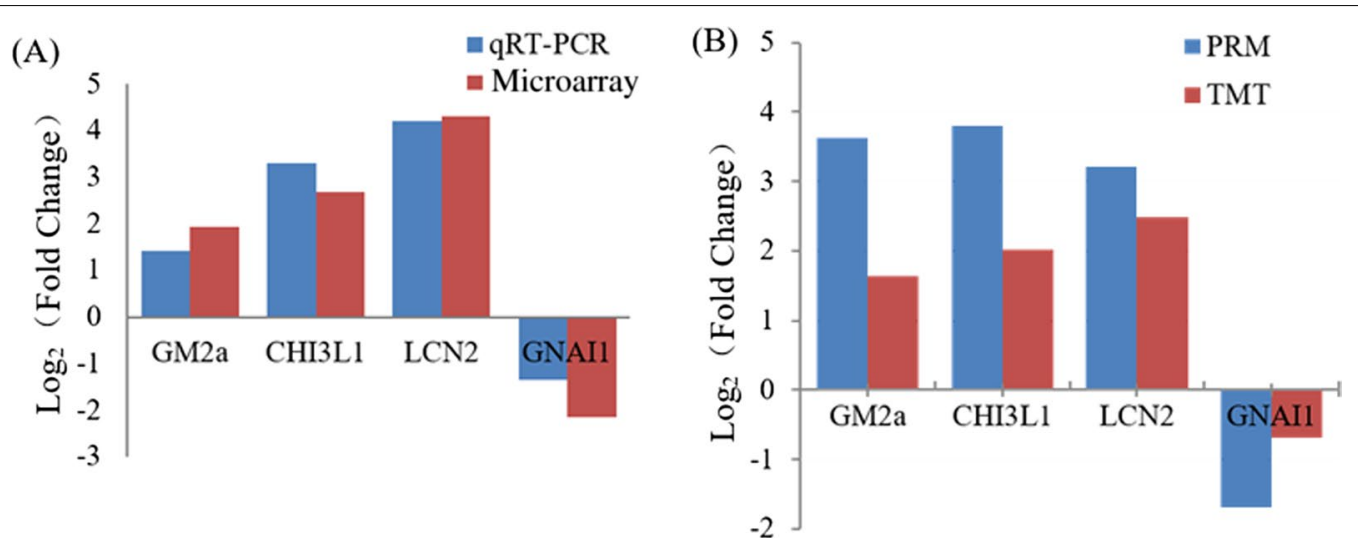

Fig. 6 Data verified by RT-qPCR and PRM. A Comparison of the expressions of DEGs determined by microarray and qRT-PCR. log2 (Fold change) of the selected DEGs measured by microarray and qRT-PCR. GM2a: GM2 ganglioside activator; CHI3L1: Chitinase-3-like protein 1; LCN2: Neutrophil gelatinase-associated lipocalin; GNAl1: Guanine nucleotide-binding protein G(i) subunit alpha-1; The transcript expression levels of the selected genes were each normalized to that of the ACTB gene. B Comparison of the quantification results between TMT and PRM. log2 (Fold change) of the DEPS were measured by TMT and PRM 
revealed that the ROS generated by NADPH oxidase is important contributor to the pathological progression of fibrotic diseases in several organs, such as the heart, lung, liver and kidney [21, 22]. ROS accumulation can induce MAPK activation [20] and secretion of TGF- $\beta$ further driving lung fibrogenesis [23]. NOX1, NOX2 and NOX4 play critical roles in initiating fibrosis through activation of hepatic stellate cells [24-26]. NOX2, the central phagocyte oxidase, locates in the plasma membrane and the membrane of intracellular vesicles of phagocytic leukocytes, such as neutrophilic granulocytes, eosinophilic granulocytes, monocytes and macrophages [27]. NOX2 needs $\mathrm{p} 22^{\text {phox }}$ to form a stable heterodimer. During phagocytosis or at sites of inflammation, a complex of three cytosolic proteins $\mathrm{p} 47^{\text {phox }}, \mathrm{p} 67^{\text {phox }}$ and $\mathrm{p} 40^{\text {phox }}$ moves to the NOX2/p22 $2^{\text {phox }}$ to start the enzymatic activity of the oxidase. In the present study, lungs of rat model of silicosis showed increased phagocyte NADPH oxidase activity with upregulated five structural components NOX2 $(C Y B B), \mathrm{p} 22^{\text {phox }}(C Y B A), \mathrm{p} 47^{\text {phox }}(N C F 1), \mathrm{p} 67^{\text {phox }}$ (NCF2) and $\mathrm{p} 40^{\text {phox }}(N C F 4)$. ROS produced mainly by phagocytes NADPH oxidase plays an important role in silica-induced pulmonary fibrosis of rats. Highly specific NOX2 inhibitors may have potential to inhibit the development of silicosis.

The vacuolar-ATPase is an important $\mathrm{pH}$ regulatory complex using the energy produced by ATP hydrolysis to pump protons into the extracellular environment. The low extracellular $\mathrm{pH}$ may promote the degradation and remolding of ECM by increasing the activity of proteolytic enzyme such as matrix metalloproteinases (MMPs) and cathepsins $[28,29]$. We found that the subunits of the vacuolar (v)-ATPase complex such as Atp6v0c, Atp6v1a, Atp6v1b2 and Atp6v1c1 were upregulated. Four cathepsins (CTSB, CTSD, CTSZ and CTSS) and MMP-9 were also upregulated. CTSS, one of the most potent elastases, can degrade fibrillar collagens, fibronectin and laminin. CTSB degrades collagen type IV, $\mathrm{X}$ and fibronectin [30]. CTSB/D can enhance expression of fibrogenic markers $\alpha$-smooth muscle actin ( $\alpha$-SMA), TGF- $\beta$ and Colla1 in hepatic stellate cells (HSCs) from ASMase-null mice. Overexpression of CTSB was found to increase hepatic fibrosis in ASMase-null mice [31]. Low extracellular $\mathrm{pH}$ caused by enhanced V-ATPase activity could activate MMP-9 and cathepsins to promote the pulmonary fibrosis. In addition, the activity of MMP-9 is regulated by Lipocalin 2 (LCN2) by forming a macromolecular LCN2/ MMP-9 complex, which protects MMP-9 from autodegradation $[32,33]$. In particular, when bound to MMP-9, LCN2 may modulate various inflammatory mechanisms including acute phase proteins and the vascular repair process [34]. LCN2 and MMP-9 have been reported to participate in pulmonary fibrosis development [35].
Chitinase 3-like 1 (CHI3L1) plays a protective role in tissue injury by ameliorating inflammation and cell death. It also has a profibrotic role in the repair phase by augmenting alternative macrophage activation, fibroblast proliferation and matrix deposition [36]. In bleomycininduced IPF the expression of CHI3L1 was found to be decreased during the early injury phase and increased during fibroproliferative repair with increasing of matrix gene expression, collagen accumulation, a-SMA expression and secretion of TGF- $\beta 1$. CHI3L1 can also interact with lung ECM to augment fibroblast proliferation and transformation to activated $[37,38]$. CHI3L1 can also regulate liver cancer potentially by regulating the TGF- $\beta$ signaling pathways with activating kinase to phosphorylate SMAD2 and SMAD3 [39]. In addition, Gm2a can cause transcription and immune responses to a range of inflammatory factors such as IL- 6 , TNF- $\alpha$ and TGF$\beta$, which can promote fibrosis by activating NF-kB signal pathway [40, 41]. CD14, Toll-like receptor 2 and macrophage receptor with collagenous structure, could interact with cord factor to promote pro-inflammatory cytokine expression [42]. An analysis of pathways shows that these pathways are interrelated and converged at common points to produce an effect (Fig. 7).

Silicosis is an occupational disease characterized as inflammatory cells infiltration and progressive pulmonary fibrosis. In this study we analyzed the proteomic/ transcriptomic profile in the lungs of rats on the 28th day after silica exposure when lung fibrosis has been formed according to our previous study. We could not provide the dynamic proteome changes occurring during silicosis. The function of these identified cor-DEG-DEP genes have not yet been explored. Further investigation is required to explore the roles of these identified cor-DEGDEP genes in silicosis.

\section{Conclusions}

In this study, we carried out a combined transcriptomic and proteomic analysis to study the molecular mechanisms underlying of pulmonary fibrosis in silica-exposed rats. Using joint analysis of transcriptomic and proteomic data we identified $250 \mathrm{DEPs} / \mathrm{DEGs}$ that mainly involve in phagosome, leukocyte transendothelial migration, complement and coagulation cascades and cell adhesion molecules (CAMs) pathways. These genes and their protein products could be critically important in initiation, progression and development of silicosis. Based on the results, we constructed the interaction map of these pathways to help understanding the molecular mechanism of silicosis (Fig. 7). Conclusively, this study provides important insights into molecular pathogenesis of silicosis and helpful information for more targeted prevention and treatment of the disease. 


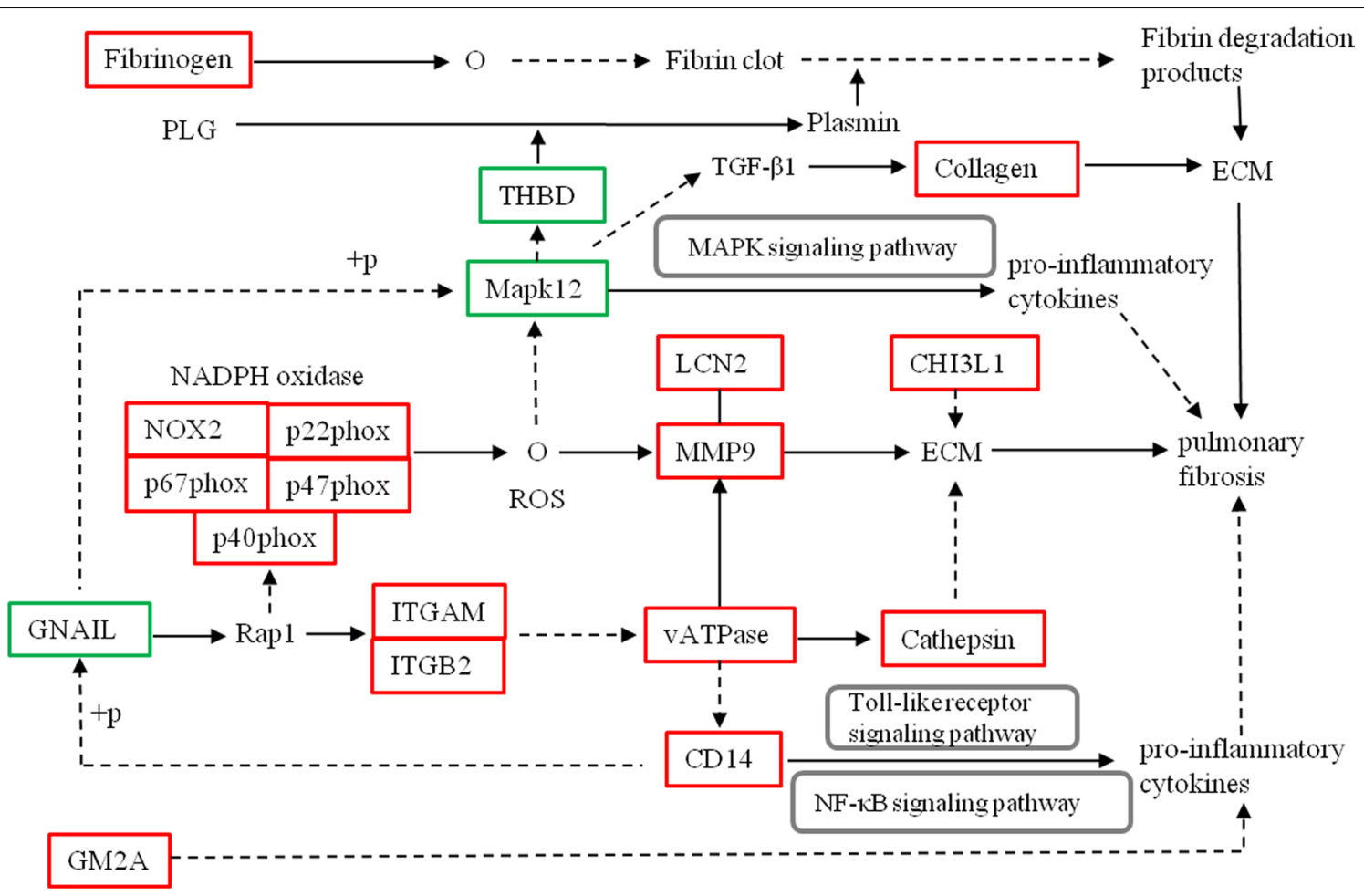

Fig. 7 Inferred mechanisms of action of the cor-DEPs-DEGs genes with mainly enrichment pathways in the silica-treated rats. Red frame: upregulated; Green frame: downregulated; $\rightarrow$ : activate or leads to; $\cdots$ : indirect effect: $\mathbf{E}$ : complexes.—: combine; + p: phosphorylation; O: chemical molecules. For simplicity in presentation, only protein designations are shown

\section{Abbreviations}

TMT:Tandem mass tags; GO: Gene Ontology; KEGG: Kyoto Encyclopedia of Genes; PRM: Parallel reaction monitoring; DEGs: Differentially expressed genes; DEPs: Differentially expressed proteins; CAMs: Cell adhesion molecules; ECM: Extracellular matrix; BP: Biological process; CC: Cellular component; MF: Molecular function; GM2a: GM2 ganglioside activator; CHI3L 1: Chitinase-3-like protein 1; LCN2: Neutrophil gelatinase-associated lipocalin; GNAl1: Guanine nucleotide-binding protein G(i) subunit alpha-1; NOXs: NADPH oxidase; IPF: Idiopathic pulmonary fibrosis; THBD: Thrombomodulin; MMPs: Matrix metalloproteinases; CHI3L1: Chitinase 3-like 1

\section{Supplementary Information}

The online version contains supplementary material available at https://doi. org/10.1186/s12890-021-01807-w.

Additional file 1: Table S1. The differentially expressed genes (DEGs) were expressed between the model and control groups.

Additional file 2: Table S2. The differentially expressed proteins (DEPS) were expressed between the model and control groups.

Additional file 3: Table S3. The cor-DEGs-DEPs genes with the same or opposite trend.

Additional file 4: Table S4. GO terms were highly enriched at both mRNA and protein levels.

Additional file 5: Table S5. KEGG terms were highly enriched at both mRNA and protein levels.
Acknowledgements

Not applicable.

\section{Authors' contributions}

SH and JQ designed research; BCX and ZJ conducted research; YGC and SLL analyzed data; BCX wrote the frst draft of manuscript; JQ and PC reviewed and edited the manuscript. All authors read and approved the final manuscript.

\section{Funding}

This work was supported by the Innovation Project of Shandong Academy of Medical Sciences, Academic promotion programme of Shandong First Medical University (2019QL001), Ji Nan Science and Technology Bureau (202019161, 201907024), Health Commission of Shandong Province (202012040412), China Coal Miner Pneumoconiosis Prevention Treatment Foundation (201915J039), Ministry of Science and Technology of PRC (2018ZX09711001-011), Natural Science Foundation of Shandong (ZR2017YL001), The Department of Science and Technology of Shandong Province (2018GSF118212), Tsung Cho Chang Education Foundation, Taiwan (UQ: 023142). We thank OeBiotech Corporation (Shanghai, China) for supporting the high throughput techniques.

\section{Availability of data and materials}

The proteomic information including the raw data, peak list files etc., have been submitted to the PRIDE database with identifier PXD020625. The microarray gene expression data have been deposited in NCBI's Gene Expression Omnibus (GEO; http://www.ncbi.nlm.nih.gov/geo/) under accession number GSE188520. 


\section{Declarations}

\section{Ethics approval and consent to participate}

All experiments were performed in accordance with the National Institutes of Health guidelines for care and use of animals and approved by the Committee on the Ethics of Animal Experiments of Shandong Academy of Occupational Health and Occupational Medicine (Protocol Number: 20190003). Additionally, all animals were handled humanely during the study protocol and during euthanasia. We confirmed this study is reported in accordance with ARRIVE guidelines.

\section{Consent for publication}

Not applicable.

\section{Competing interests}

The authors declare that they have no competing interests.

\section{Author details}

'Shandong Academy of Occupational Health and Occupational Medicine, Shandong First Medical University \& Shandong Academy of Medical Sciences, Ji'nan, Shandong, China. ${ }^{2}$ Queensland Alliance for Environmental Health Sciences, The University of Queensland, Brisbane, QLD, Australia.

Received: 13 May 2021 Accepted: 13 December 2021

Published online: 07 January 2022

\section{References}

1. Nardi J, Nascimento S, Göethel G, Gauer B, Sauer E, Fão N, et al. Inflammatory and oxidative stress parameters as potential early biomarkers for silicosis. Clin Chim Acta. 2018;484:305-13. https://doi.org/10.1016/j.cca. 2018.05.045.

2. Yang J, Wang T, Li Y, Yao W, Ji X, Wu Q, et al. Earthworm extract attenuates silica-induced pulmonary fibrosis through Nrf2-dependent mechanisms. Lab Investig. 2016;96(12):1279-300. https://doi.org/10.1038/labinvest. 2016.101

3. Han R, Ji X, Rong R, Li Y, Yao W, Yuan J, et al. MiR-449a regulates autophagy to inhibit silica-induced pulmonary fibrosis through targeting $\mathrm{BCl} 2$. J Mol Med (Berl). 2016;94(11):1267-79. https://doi.org/10.1007/ s00109-016-1441-0.

4. Du HW, Chen GY, Yang CH, Li CB, Xun YP, Liu J, et al. Multiple correlations of mRNA expression and protein abundance in human cytokine profile. Mol Biol Rep. 2014;41(10):6985-93. https://doi.org/10.1007/ s11033-014-3585-8

5. Wettersten $\mathrm{HI}$, Hakimi AA, Morin D, Bianchi C, Johnstone ME, Donohoe DR, et al. Grade-dependent metabolic reprogramming in kidney cancer revealed by combined proteomics and metabolomics analysis. Cancer Res. 2015;75(12):2541-52. https://doi.org/10.1158/0008-5472. CAN-14-1703.

6. Raherison E, Rigault P, Caron S, Poulin PL, Boyle B, Verta JP, et al. Transcriptome profiling in conifers and the PiceaGenExpress database show patterns of diversification within gene families and interspecific conservation in vascular gene expression. BMC Genomics. 2012;13:434. https://doi. org/10.1186/1471-2164-13-434

7. Qiu Z, Wan L, Chen T, Wan Y, He X, Lu S, et al. The regulation of cambial activity in Chinese fir (Cunninghamia lanceolata) involves extensive transcriptome remodeling. New Phytol. 2013;199(3):708-19. https://doi. org/10.1111/nph.12301

8. Suhre K, McCarthy MI, Schwenk JM. Genetics meets proteomics: perspectives for large population-based studies. Nat Rev Genet. 2021:22(1):19-37. https://doi.org/10.1038/s41576-020-0268-2.

9. Bo C, Geng X, Zhang J, Sai L, Zhang Y, Yu G, et al. Comparative proteomic analysis of silica-induced pulmonary fibrosis in rats based on tandem mass tag (TMT) quantitation technology. PLoS ONE. 2020;15(10):e0241310. https://doi.org/10.1371/journal.pone.0241310.

10. Livak KJ, Schmittgen TD. Analysis of relative gene expression data using 453 real-time quantitative PCR and the 2(-Delta Delta $C(T))$ method. Methods. 2001;25(4):402-8.
11. Pascovici D, Handler DC, Wu JX, Haynes PA. Multiple testing corrections in quantitative proteomics: a useful but blunt tool. Proteomics. 2016;16(18):2448-53. https://doi.org/10.1002/pmic.201600044.

12. Wang $X$, Li D, Song S, Zhang Y, Li Y, Wang $X$, et al. Combined transcriptom ics and proteomics forecast analysis for potential genes regulating the Columbian plumage color in chickens. PLoS ONE. 2019;14(11):e0210850. https://doi.org/10.1371/journal.pone.0210850.

13. Yan J, Yang $X$, Jiao $X$, Yang $X$, Guo $M$, Chen $Y$, et al. Integrative transcriptomic and proteomic analysis reveals CD9/ITGA4/PI3K-Akt axis mediates trabecular meshwork cell apoptosis in human glaucoma. J Cell Mol Med. 2020;24(1):814-29. https://doi.org/10.1111/jcmm.14792.

14. Fang CC, Huang JW, Shyu RS, Yen CJ, Shiao CH, Chiang CK, et al. Fibrininduced epithelial-to-mesenchymal transition of peritoneal mesothelial cells as a mechanism of peritoneal fibrosis: effects of pentoxifylline. PLoS ONE. 2012;7(9):e44765. https://doi.org/10.1371/journal.pone.0044765.

15. Fujimoto H, Gabazza EC, Taguchi O, Nishii Y, Nakahara H, Bruno NE, et al. Thrombin-activatable fibrinolysis inhibitor deficiency attenuates bleomycin-induced lung fibrosis. Am J Pathol. 2006;168(4):1086-96. https://doi. org/10.2353/ajpath.2006.050610.

16. Chambers RC. Abnormal wound healing responses in pulmonary fibrosis: focus on coagulation signalling. Eur Respir Rev. 2008;17(109):130-7.

17. Wygrecka M, Markart P, Ruppert C, Petri K, Preissner KT, Seeger W, et al. Cellular origin of pro-coagulant and (anti)-fibrinolytic factors in bleomycin-injured lungs. Eur Respir J. 2007;29(6):1105-14. https://doi. org/10.1183/09031936.00097306.

18. Bargagli E, Madioni C, Bianchi N, Refini RM, Cappelli R, Rottoli P. Serum analysis of coagulation factors in IPF and NSIP. Inflammation. 2014;37(1):10-6. https://doi.org/10.1007/s10753-013-9706-z.

19. Delvaeye M, Noris M, De Vriese A, Esmon CT, Esmon NL, Ferrell G, et al. Thrombomodulin mutations in atypical haemolytic-uremic syndrome. N Engl J Med. 2009;361(4):345-57. https://doi.org/10.1056/NEJMoa0810 739.

20. Wang D, Yan Z, Bu L, An C, Deng B, Zhang J, et al. Protective effect of peptide DR8 on bleomycin-induced pulmonary fibrosis by regulating the TGF- $\beta / M A P K$ signaling pathway and oxidative stress. Toxicol Appl Pharmacol. 2019;382: 114703. https://doi.org/10.1016/j.taap.2019.114703.

21. Hecker L, Cheng J, Thannickal VJ. Targeting NOX enzymes in pulmonary fibrosis. Cell Mol Life Sci. 2012;69(14):2365-71. https://doi.org/10.1007/ s00018-012-1012-7.

22. Sedeek M, Nasrallah R, Touyz RM, Hébert RL. NADPH oxidases, reactive oxygen species, and the kidney: friend and foe. J Am Soc Nephrol. 2013:24(10):1512-8. https://doi.org/10.1681/ASN.2012111112.

23. Gorowiec MR, Borthwick LA, Parker SM, Kirby JA, Saretzki GC, Fisher AJ. Free radical generation induces epithelial-to-mesenchymal transition in lung epithelium via a TGF- $\beta 1$-dependent mechanism. Free Radic Biol Med. 2012;52(6):1024-32. https://doi.org/10.1016/j.freeradbiomed.2011 12.020 .

24. Paik YH, Iwaisako K, Seki E, Inokuchi S, Schnabl B, Osterreicher $\mathrm{CH}$, et al. The nicotinamide adenine dinucleotide phosphate oxidase (NOX) homologues NOX1 and NOX2/gp91 (phox) mediate hepatic fibrosis in mice. Hepatology. 2011:53(5):1730-41. https://doi.org/10.1002/hep.24281.

25. Jiang JX, Chen X, Serizawa N, Szyndralewiez C, Page P, Schröder K, et al. Liver fibrosis and hepatocyte apoptosis are attenuated by GKT137831, a novel NOX4/NOX1 inhibitor in vivo. Free Radic Biol Med. 2012;53(2):28996. https://doi.org/10.1016/j.freeradbiomed.2012.05.007.

26. Sancho P, Mainez J, Crosas-Molist E, Roncero C, Fernández-Rodriguez CM, Pinedo $F$, et al. NADPH oxidase NOX4 mediates stellate cell activation and hepatocyte cell death during liver fibrosis development. PLOS ONE. 2012;7(9):e45285. https://doi.org/10.1371/journal.pone.0045285.

27. Nunes P, Demaurex N, Dinauer MC. Regulation of the NADPH oxidase and associated ion fluxes during phagocytosis. Traffic. 2013;14(11):1118-31. https://doi.org/10.1111/tra.12115.

28. Fan SH, Wang YY, Lu J, Zheng YL, Wu DM, Zhang ZF, et al. CERS2 suppresses tumor cell invasion and is associated with decreased V-ATPase and MMP-2/MMP-9 activities in breast cancer. J Cell Biochem. 2015;116(4):502-13. https://doi.org/10.1002/jcb.24978.

29. Fais S, De Milito A, You H, Qin W. Targeting vacuolar H+-ATPases as a new strategy against cancer. Cancer Res. 2007;67(22):10627-30. https://doi. org/10.1158/0008-5472.CAN-07-1805. 
30. Martin SL, Moffitt KL, McDowell A, Greenan C, Bright-Thomas RJ, Jones $A M$, et al. Association of airway cathepsin $B$ and $S$ with inflammation in cystic fibrosis. Pediatr Pulmonol. 2010;45(9):860-8. https://doi.org/10, 1002/ppul.21274.

31. Moles A, Tarrats N, Fernández-Checa JC, Marí M. Cathepsin B overexpression due to acid sphingomyelinase ablation promotes liver fibrosis in Niemann-Pick disease. J Biol Chem. 2012;287(2):1178-88. https://doi.org/ 10.1074/jbc.M111.272393.

32. Volpe V, Raia Z, Sanguigno L, Somma D, Mastrovito P, Moscato F, et al. NGAL controls the metastatic potential of anaplastic thyroid carcinoma cells. J Clin Endocrinol Metab. 2013;98(1):228-35. https://doi.org/10.1210/ jc.2012-2528.

33. Berger T, Cheung CC, Elia AJ, MakTW. Disruption of the Lcn2 gene in mice suppresses primary mammary tumor formation but does not decrease lung metastasis. Proc Natl Acad Sci U S A. 2010;107(7):2995-3000. https:// doi.org/10.1073/pnas.1000101107.

34. Capoluongo E, Vento G, Lulli P, Di Stasio E, Porzio S, Vendettuoli V, et al. Epithelial lining fluid neutrophil-gelatinase-associated lipocalin levels in premature newborns with bronchopulmonary dysplasia and patency of ductus arteriosus. Int J Immunopathol Pharmacol. 2008;21 (1):173-9. https://doi.org/10.1177/039463200802100119.

35. Han X, Liu H, Zhang Z, Yang W, Wu C, Liu X, et al. Epitranscriptomic 5-methylcytosine profile in $\mathrm{PM}_{2.5}$-induced mouse pulmonary fibrosis. Genomics Proteomics Bioinform. 2020;18(1):41-51. https://doi.org/10. 1016/j.gpb.2019.11.005.

36. Recklies AD, White $\mathrm{C}$, Ling $\mathrm{H}$. The chitinase 3-like protein human cartilage glycoprotein 39 (HC-gp39) stimulates proliferation of human connective-tissue cells and activates both extracellular signal-regulated kinase- and protein kinase B-mediated signalling pathways. Biochem J. 2002;365(Pt1):119-26. https://doi.org/10.1042/BJ20020075.

37. Lee CG, Hartl D, Lee GR, Koller B, Matsuura H, Da Silva CA, et al. Role of breast regression protein 39 (BRP-39)/chitinase 3-like-1 in Th2 and IL13-induced tissue responses and apoptosis. J Exp Med. 2009;206(5):114966. https://doi.org/10.1084/jem.20081271.

38. Zhou Y, Peng H, Sun H, Peng X, Tang C, Gan Y, et al. Chitinase 3-like 1 suppresses injury and promotes fibroproliferative responses in Mammalian lung fibrosis. Sci Transl Med. 2014;6(240):240ra76. https://doi.org/10.1126/ scitranslmed.3007096.

39. Qiu QC, Wang L, Jin SS, Liu GF, Liu J, Ma L, et al. CHI3L1 promotes tumor progression by activating TGF- $\beta$ signaling pathway in hepatocellular carcinoma. Sci Rep. 2018;8(1):15029. https://doi.org/10.1038/ s41598-018-33239-8.

40. Maghsoudloo M, Azimzadeh Jamalkandi S, Najafi A, Masoudi-Nejad A. Identification of biomarkers in common chronic lung diseases by co-expression networks and drug-target interactions analysis. Mol Med. 2020;26(1):9. https://doi.org/10.1186/s10020-019-0135-9.

41. Caplan MS, Simon D, Jilling T. The role of PAF, TLR, and the inflammatory response in neonatal necrotizing enterocolitis. Semin Pediatr Surg. 2005;14(3):145-51. https://doi.org/10.1053/j.sempedsurg.2005.05.002.

42. Roedig H, Nastase MV, Frey H, Moreth K, Zeng-Brouwers J, Poluzzi C, et al. Biglycan is a new high-affinity ligand for CD14 in macrophages. Matrix Biol. 2019;77:4-22. https://doi.org/10.1016/j.matbio.2018.05.006.

\section{Publisher's Note}

Springer Nature remains neutral with regard to jurisdictional claims in published maps and institutional affiliations.

Ready to submit your research? Choose BMC and benefit from:

- fast, convenient online submission

- thorough peer review by experienced researchers in your field

- rapid publication on acceptance

- support for research data, including large and complex data types

- gold Open Access which fosters wider collaboration and increased citations

- maximum visibility for your research: over $100 \mathrm{M}$ website views per year

At BMC, research is always in progress.

Learn more biomedcentral.com/submissions 